\title{
Spinal leptin contributes to the development of morphine antinociceptive tolerance by activating the STAT3-NMDA receptor pathway in rats
}

\author{
FEN HU $^{1}$, YU CUI ${ }^{1}$, RUIXIAN GUO ${ }^{1}$, JINGFU CHEN $^{2}$, RUNMING GUO ${ }^{2}$, NING SHEN ${ }^{1}$, \\ XIAOXIAO HUA ${ }^{3}$, LIQIU $\mathrm{MO}^{3}$ and JIANQIANG FENG ${ }^{1}$ \\ ${ }^{1}$ Department of Physiology, Zhongshan School of Medicine, Sun Yat-sen University, Guangzhou, Guangdong 510080; \\ ${ }^{2}$ Department of Cardiovasology, The Affiliated Hospital, Guangdong Medical College, Zhanjiang, \\ Guangdong 524001; ${ }^{3}$ Department of Anesthesiology, Huangpu Division of The First Affiliated \\ Hospital, Sun Yat-sen University, Guangzhou, Guangdong 510700, P.R. China
}

Received August 10,2013; Accepted April 1, 2014

DOI: $10.3892 / \mathrm{mmr} .2014 .2250$

\begin{abstract}
Leptin, an adipokine synthesized mainly by non-neuronal tissues, has been reported to contribute to the pathogenesis of neuropathic pain. It has been hypothesized that morphine tolerance and neuropathic pain share some common pathological mechanisms. The present study was designed to examine whether spinal leptin is implicated in the development of morphine antinociceptive tolerance, and whether spinal leptin induces the activation of signal transducer and activator of transcription 3 (STAT3) signaling pathway and the NR1 subunit of N-methyl-D-aspartate (NMDA) receptor, in morphine antinociceptive tolerance in rats. The results demonstrated that intrathecal (i.t.) administration of a leptin antagonist (LA) prevented the development of morphine antinociceptive tolerance in rats. Further studies revealed that the levels of the spinal leptin and the leptin receptor (Ob-R) were time-dependently increased following chronic morphine treatment. Mechanistic examination indicated that chronic morphine triggered activation of the STAT3 pathway and an increase in the expression of the NR1 subunit of the NMDA receptor, which was ameliorated by i.t. administration of AG490 [a Janus kinase (JAK)-STAT inhibitor]. The increased activation of STAT3 and the NR1 subunit was markedly attenuated by i.t. treatment with LA. In addition, the spinal administration of AG490 or MK-801 (a
\end{abstract}

Correspondence to: Dr Liqiu Mo, Department of Anesthesiology, Huangpu Division of The First Affiliated Hospital, Sun Yat-sen University, No. 74, Zhongshan Road 2, Guangzhou, Guangdong 510700, P.R. China

E-mail: mlqiu11220@126.com

Professor Jianqiang Feng, Department of Physiology, Zhongshan School of Medicine, Sun Yat-sen University, No 74, Zhongshan Road 2, Guangzhou, Guangdong 510080, P.R. China

E-mail: fengjq-sums@163.com

Key words: morphine antinociceptive tolerance, leptin, STAT3, NMDA receptor, spinal cord non-competitive NMDA receptor inhibitor) blocked the development of morphine antinociceptive tolerance. Taken together, these results have demonstrated, for the first time, to the best of our knowledge, that spinal leptin contributes to the development of morphine antinociceptive tolerance by activating the spinal STAT3-NMDA receptor pathway.

\section{Introduction}

Opioids, such as morphine, have been widely utilized clinically as one of the most potent analgesics for treating acute and chronic pain conditions. However, the clinical utility of opioid analgesics is often limited by the development of analgesic tolerance, that necessitates dose escalation. An increasing number of studies are investigating the neurobiological underpinnings of opioid tolerance, including those on N-methyl-D-aspartate (NMDA) receptors $(1,2)$, proinflammatory cytokines [interleukin-1 $\beta$ (IL-1 $\beta)$, IL-6 and tumor necrosis factor- $\alpha$ (TNF- $\alpha)](3,4)$ and signal transducer and activator of transcription 3 (STAT3) (5). Recently, we have demonstrated the role of p38 mitogen-activated protein kinase (MAPK) in the development of morphine antinociceptive tolerance $(6,7)$. Based on the data from these studies, it was proposed that the molecules, which can modulate elements such as NMDA receptors, IL-1 $\beta$, p38 MAPK and STAT3, may contribute to the development of morphine antinociceptive tolerance. It was hypothesized that one candidate molecule for this may be leptin.

Leptin, an adipokine, is a $16 \mathrm{kDa}$, nonglycosylated peptide hormone encoded by the obese gene (ob) in mice (8). Stimulation of its receptor leads to activation of the Janus kinase (JAK)-STAT signaling pathway (9). Numerous studies have revealed that leptin is crucial in nociceptive behavior elicited by nerve injury in rats $(10,11)$. While the peripheral effect of leptin on neuropathic pain is mediated by macrophage stimulation (10), its central effect is likely associated with the upregulation of NMDA receptors following nerve injury (11). A more recent study also reported that intrathecal (i.t.) leptin enhances the expression of NMDA receptors and phosphorylated (p)-STAT3 within the rat spinal cord dorsal horn (12). Since it was hypothesized that 
morphine tolerance and neuropathic pain share certain common pathological mechanisms $(13,14)$, the present study investigated the hypothesis that (i) spinal leptin would be involved in the development of morphine antinociceptive tolerance in rats and (ii) the proposed spinal leptin effect would be mediated by leptin-dependent downstream cellular responses, including the increased expression of spinal STAT3 and NMDA receptors. The results demonstrated that chronic i.t. administration of morphine significantly upregulates the expression levels of spinal leptin, leptin receptor (Ob-R) and p-STAT3, as well as the NR1 subunit of NMDA receptors in the spinal cord. The data suggest that the activation of spinal STAT3-NMDA receptor pathway contributes to leptin-mediated development of morphine antinociceptive tolerance in rats.

\section{Materials and methods}

Materials. Morphine hydrochloride was purchased from Qinghai Pharmaceutical Factory Co., Ltd. (Xining, Qinghai, China). Leptin antagonist (LA) was obtained from ProSpec-Tany TechnoGene Ltd. (East Brunswick, NJ, USA). AG490 and MK-801 were purchased from Sigma-Aldrich (St. Louis, MO, USA).

Animals. Adult male Sprague-Dawley rats (weight, 250-280 g) were provided by Sun Yat-sen University Experimental Animal Center (Guangzhou, Guangdong, China) and housed individually with free access to water and food at a temperature of $22 \pm 2^{\circ} \mathrm{C}$ and in a light control (12 h light-dark cycle) quiet room. All experimental procedures were approved by the ethics committee of Zhongshan School of Medicine, Sun Yat-sen University (Guangzhou, China) and were conducted strictly in compliance with the NIH guidelines for the Care and Use of Laboratory Animals.

I.t. catheter implantation. For i.t. delivery of the indicated drugs (such as morphine, etc.), rats were implanted with i.t. catheters according to the methods described previously (6). Briefly, a sterile polyethylene $(\mathrm{PE}-10)$ tube (length=7 $\mathrm{cm}$ ) filled with saline was inserted through the L5/L6 intervertebral space and the tip of the tube was placed at the spinal lumbar enlargement level. Correct i.t. catheter placement was verified following the completion of behavioral test by visual inspection. The wound was closed in two layers with 4-0 polyester suture. All the animals were allowed to recover for at least 4 days prior to the experiments. Any rats that developed hind limb paralysis or paresis following surgery were excluded and euthanized with an overdose of pentobarbital.

Drug administration. Drugs were administered in volumes of $10 \mu \mathrm{l}$ followed by a flush of $7 \mu \mathrm{l}$ of saline to ensure drug delivery into the subarachnoid space via the i.t. catheter. Morphine hydrochloride was dissolved in saline. According to the previously described methods, LA (3 $\mu \mathrm{g})$ (11), AG490 (1 $\mu \mathrm{g})(11)$ and MK-801 (10 nmol) (12) were dissolved in DMSO and diluted by saline to a final volume of $10 \mu \mathrm{l}$. The residual drug solution was discarded following each experiment.

Induction of morphine antinociceptive tolerance and behavioral test. Tolerance to morphine antinociceptive effect was induced by i.t. administration of morphine (15 $\mu \mathrm{g}$ daily) for 7 days. Nociceptive tests were performed prior to and $30 \mathrm{~min}$ following morphine administration on day $1,3,5$ and 7 . To examine the effect of LA, AG490 or MK-801 on morphine antinociceptive tolerance, drugs were administered via i.t. $30 \mathrm{~min}$ prior to each morphine administration, respectively.

Morphine antinociception was evaluated by the hot-water tail-flick test according to the previously described methods (6). Briefly, rats were restrained in a plastic container $(22 \times 6 \mathrm{~cm})$ and the distal third of the tail was immersed into the water maintained at $50 \pm 0.2^{\circ} \mathrm{C}$. The latency response was defined by rapid removal of the tail from the water. A cut off time of $15 \mathrm{sec}$ was used to avoid tissue damage. Three trials were performed for each rat with an intertrial interval of $2 \mathrm{~min}$ and the mean of three measurements was obtained as the final latency. The percentage of maximal possible antinociceptive effect (\%MPE) was calculated by comparing the test latency prior to [baseline (BL)] and post-drug injection (TL) using the equation: \%MPE $=[(\mathrm{TL}-\mathrm{BL}) /($ cut off time-BL $)] \times 100 \%$.

Immunohistochemistry. Immediately following the behavioral test on the indicated days, rats were deeply anesthetized with sodium pentobarbital (100 mg/kg, i.p.), and perfused through the ascending aorta with cold saline, followed by $4 \%$ paraformaldehyde in 0.1 M PBS ( $\left.\mathrm{pH} \mathrm{7.2-7.4,} 4^{\circ} \mathrm{C}\right)$. Following perfusion, the spinal cord lumbar enlargement was quickly removed and post-fixed in the same fixative for $2 \mathrm{~h}$ and then cytoprotected in $30 \%$ sucrose for two nights. Transverse spinal sections $(20 \mu \mathrm{m})$ were cut in a cryostat, mounted on polylysine-coated slides and processed for immunohistochemistry. All of the sections were blocked with $2 \%$ goat or donkey serum in $0.1 \mathrm{M} \mathrm{TBS} / 0.3 \%$ Triton X-100 for $1 \mathrm{~h}$ at room temperature (RT) and incubated over two nights at $4^{\circ} \mathrm{C}$ with the primary antibody for leptin (Ob; dilution, 1:100), leptin receptor (Ob-R; dilution, 1:50; Santa Cruz Biotechnology, Inc., Santa Cruz, CA, USA) or p-STAT3 (Tyr705; dilution, 1:50; Cell Signaling Technology, Inc., Danvers, MA, USA), NMDA receptor NR1 subunit (NR1; dilution, 1:100; Bioworld Technology, Inc., MN, USA), glial fibrillary acidic protein (GFAP; astrocyte marker; dilution, 1:400), OX-42 (microglia marker; dilution, 1:400) or NeuN (neuron marker; dilution, 1:300; Merck Millipore, Billerica, MA, USA). For double immunofluorescent staining, the primary antibody for the protein of interest and the marker for the above cells were mixed. Then, the slides were incubated away from the light at RT for $2 \mathrm{~h}$ with the corresponding single or mixed FITC- or Cy3-conjugated secondary fluorescent antibody (dilution, 1:400; Jackson ImmunoResearch, Western Grove, PA, USA). The stained sections were observed with an Olympus fluorescence microscope (Olympus, Tokyo, Japan) and the images were captured by a CCD spot Camera. Non-specific staining was determined by omitting the primary antibodies.

Western blot assay. The lumbar enlargements of the spinal cord were removed immediately following the behavioral tests and placed into liquid nitrogen for quick freezing. The tissue was put into radioimmunoprecipitation assay (RIPA) buffer containing protease and phosphatase inhibitor cocktails (Sigma-Aldrich) to be homogenized on ice. Following centrifugation at $4^{\circ} \mathrm{C}$, the supernatants were collected. Then, 
the protein samples were separated by SDS-polyacrylamide gel electrophoresis and electrotransferred onto polyvinylidene fluoride membrane (Merck Millipore). The membrane was blocked by $5 \%$ non-fat milk for $1 \mathrm{~h}$ at RT, then incubated overnight at $4^{\circ} \mathrm{C}$ with the primary antibody for leptin $(\mathrm{Ob}$; dilution, 1:1,000) or leptin receptor (Ob-R; dilution, 1:500, Santa Cruz Biotechnology, Inc.) or p-STAT3 (Tyr705; dilution, 1:500; Cell Signaling Technology, Inc.) or NMDA receptor NR1 (NR1; dilution, 1:500; Bioworld Technology, Inc.), respectively. Then, the membrane was incubated with the horseradish peroxidase labeled secondary antibody (1:3,000; Bioworld Technology, Inc.) for $1.5 \mathrm{~h}$ at RT, developed in ECL solution and exposed onto X-ray film.

Statistical analysis. All data are presented as the mean \pm SEM. Differences between groups were analyzed by one-way analysis of variance (ANOVA) using SPSS 17.0 software and followed by LSD post hoc comparison test. $\mathrm{P}<0.05$ was considered to indicate a statistically significant result.

\section{Results}

Spinal leptin critically contributes to morphine antinociceptive tolerance in rats. To test whether the blockage of spinal leptin would prevent the development of morphine antinociceptive tolerance following chronic morphine exposure, a LA was administered via i.t. once daily for 7 days, 30 min prior to morphine treatment. This treatment regimen significantly reduced the development of morphine antinociceptive tolerance when examined at $30 \mathrm{~min}$ following morphine treatment on days 3,5 and 7, as compared with the morphine treatment group (Fig. 1). However, repeated exposure to normal saline (NS) or LA (3 $\mu \mathrm{g})$ alone did not elicit any analgesic effect as compared with the saline group assessed by the tail-flick test (Fig. 1). These results suggested that the spinal leptin is critical in the development of morphine antinociceptive tolerance.

Chronic morphine exposure induces the upregulation of spinal leptin receptor expression in rats. To examine the molecular mechanisms responsible for the spinal leptin effects on morphine antinociceptive tolerance, we first determined whether the expression of leptin receptor (Ob-R) in the spinal cord would be altered following chronic morphine treatment because $\mathrm{Ob}-\mathrm{R}$ is considered to be responsible for the majority of leptin functions $(15,16,17)$. As demonstrated by the data of the western blot assay, the expression of spinal Ob-R protein was enhanced at a time course similar to that of the development of morphine antinociceptive tolerance (Fig. 2A and B). In addition, $\mathrm{Ob}-\mathrm{R}$ immunoreactivity was topographically increased in the spinal cord dorsal horn of chronic morphine treatment rats (Fig. 2C). The spinal Ob-R immunoreactivity was colocalized primarily with NeuN (a neuronal marker) and, to a lesser extent, with GFAP (an astrocyte marker; Fig. 2D), revealing that chronic morphine exposure mainly induced the upregulation of neuronal $\mathrm{Ob}-\mathrm{R}$ in the spinal cord dorsal horn of rats.

Chronic morphine exposure enhances the spinal expression of leptin. The changes in spinal leptin expression during the development of morphine antinociceptive tolerance were

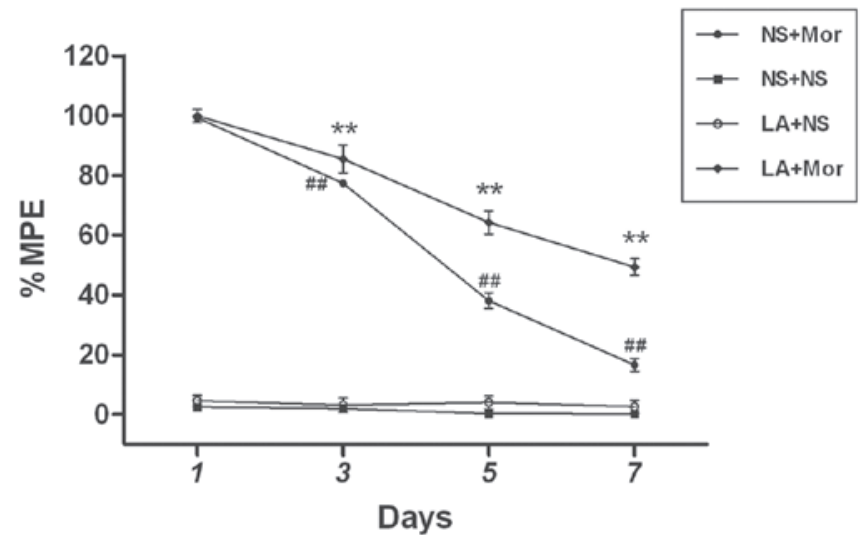

Figure 1. Effect of i.t. injection of leptin antagonist on the development of morphine antinociceptive tolerance. Mor antinociception was evaluated by hot water tail-flick test as described in Materials and methods. LA (3 $\mu \mathrm{g})$ was administered $30 \mathrm{~min}$ prior to daily Mor $(15 \mu \mathrm{g})$ i.t. injection. Data are presented as the mean \pm SEM $(n=6)$. ${ }^{* *} \mathrm{P}<0.001$ vs. (NS+Mor) group, ${ }^{\# \#} \mathrm{P}<0.001$ vs. (NS+NS) group. NS, normal saline; Mor, morphine; LA, leptin antagonist; i.t., intrathecal.

examined. As illustrated in Fig. 3, the increased spinal leptin expression (western blot analysis) on day 1 following morphine treatment was not statistically significant as compared with the saline group. By contrast, the increases in spinal leptin expression observed on days 3,5 and 7, were significantly different in the morphine-treated rats, as compared with the control group $(\mathrm{P}<0.05)$, respectively, as evidenced by a time-dependent increase in the spinal leptin expression following chronic morphine exposure.

$L A$ reduces chronic morphine-induced phosphorylation of the spinal STAT3. Thirdly, we examined whether there was a correlation between the spinal STAT3 activation and leptin, following chronic exposure to morphine. The data from the western blot analysis demonstrated that the phosphorylated expression of spinal STAT3 was markedly increased on day 7 following chronic morphine treatment as compared with the saline group ( $\mathrm{P}<0.01$; Fig. $4 \mathrm{~A}$ and $\mathrm{B})$. Of note, the increase in spinal p-STAT3 expression by chronic morphine was markedly inhibited by i.t. administration of spinal LA ( $3 \mu \mathrm{g})$, suggesting that spinal STAT3 activation is modulated by the spinal leptin following chronic morphine treatment. LA (3 $\mu \mathrm{g})$ alone did not alter the basal expression of spinal p-STAT3 (Fig. 4A and B). In addition, p-STAT3 immunoreactivity was topographically increased in the spinal dorsal horn of chronic morphine treatment rats (Fig. 4C). The spinal p-STAT3 immunoreactivity was colocalized primarily with GFAP (a marker of astrocytes; Fig. 4D).

LA attenuates chronic morphine-induced increase in the spinal NR1 subunit of NMDA receptors in rats. Following this, we examined whether there was a link between the spinal NR1 subunit expression and the spinal leptin following chronic morphine exposure. The findings of the western blot analysis demonstrated that the expression of spinal NR1 was markedly increased on day 7 following chronic morphine treatment as compared with that in the saline group $(\mathrm{P}<0.01$; Fig. 5A and B). Of note, the increased spinal NR1 expression 
A

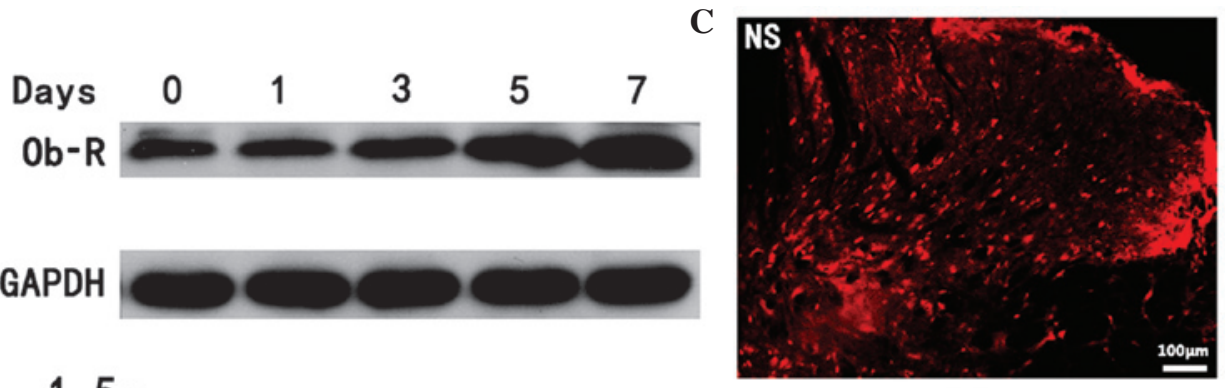

B
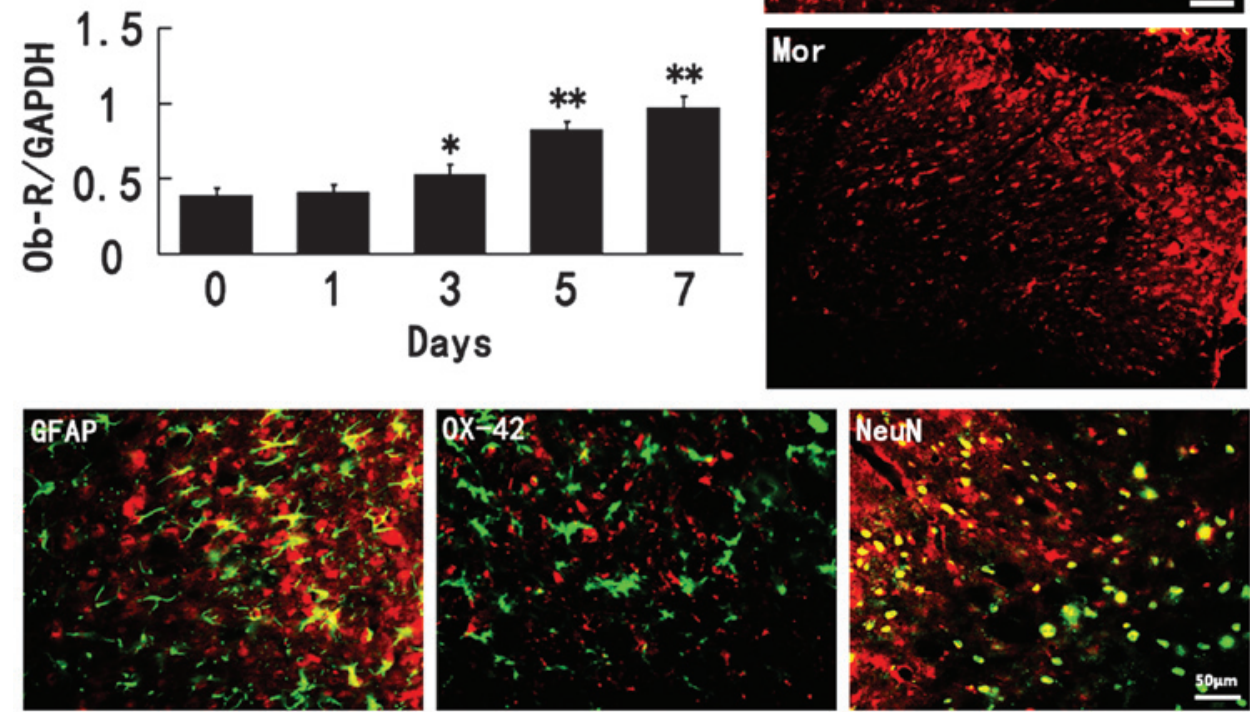

Figure 2. Chronic i.t. injection of morphine increases the expression of Ob-R of the spinal cord in rats. (A) Western blot assay demonstrates an increase in the expression of $\mathrm{Ob}-\mathrm{R}$ in the indicated days following morphine injection. (B) The data in (A) were quantified by densitometric analysis using Image J 1.41o software. (C) Immunofluorescence data revealed an increase in Ob-R IR in the spinal dorsal horn after 7 days of morphine exposure. Scale bar: $100 \mu \mathrm{m}$. (D) Double immunofluorescence data demonstrate that Ob-R was mainly co-localized with NeuN (a neuronal marker), and to a lesser extent, with GFAP (an astrocyte marker). Scale bar: $50 \mu \mathrm{m}$. Data are represented as the mean $\pm \mathrm{SEM}(\mathrm{n}=5)$. ${ }^{*} \mathrm{P}<0.01,{ }^{* *} \mathrm{P}<0.001$ vs. control group. Ob-R, leptin receptor; GFAP, glial fibrillary acidic protein; NS, normal saline; Mor, morphine; i.t., intrathecal; IR, immunoreactivity.

A

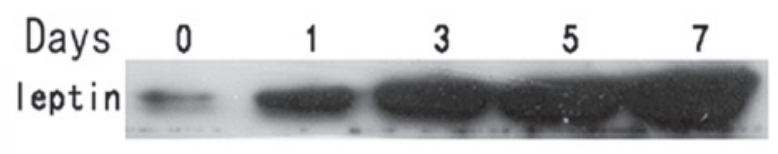

GAPDH

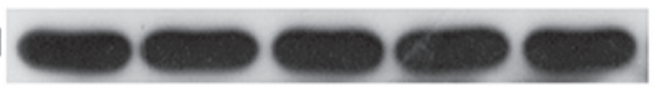

B

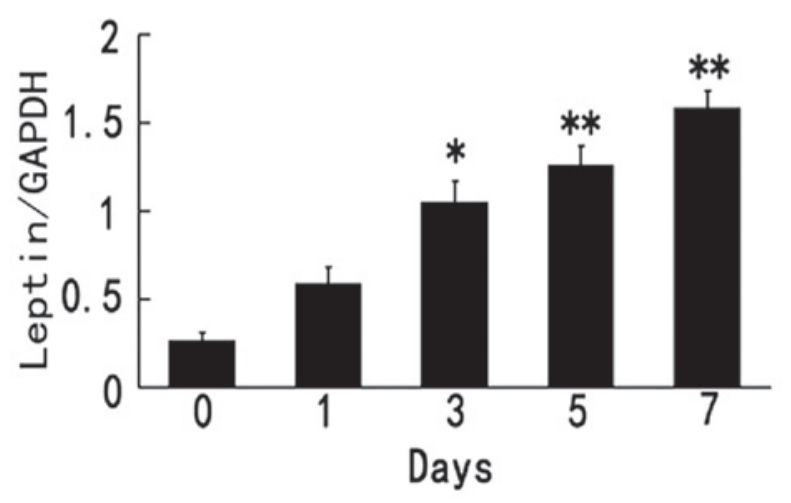

Figure 3. Chronic i.t. administration of morphine increases the expression of spinal leptin in rats. (A) Western blot analysis demonstrated the increased spinal leptin expression in the indicated times following chronic morphine treatment. (B) The data in (A) were quantified by densitometric analysis using Image J 1.41 o software. Data are represented as the mean \pm SEM $(n=3)$. ${ }^{*} \mathrm{P}<0.05,{ }^{* *} \mathrm{P}<0.01$ vs. the control on day 0 . i.t., intrathecal. induced by chronic morphine treatment was markedly inhibited by i.t. administration of LA $(3 \mu \mathrm{g})$, suggesting that the spinal NR1 expression is modulated by the spinal leptin following chronic morphine treatment. LA (3 $\mu \mathrm{g})$ alone did not alter the basal expression of spinal NR1 (Fig. 5A and B). In addition, NR1 immunoreactivity was topographically increased in the spinal cord dorsal horn in the chronic morphine treatment rats (Fig. 5C). Double immunofluorescence results revealed that the spinal NR1 immunoreactivity was colocalized primarily with NeuN (a neuronal marker; Fig. 5D).

JAK-STAT inhibitor ameliorates chronic morphine-induced upregulation of the spinal NRI subunit of NMDA receptors. Next, we observed whether the spinal STAT pathway was implicated in chronic morphine-induced increase in spinal NR1 subunit expression. As illustrated in Fig. 6, pretreatment with $1 \mu \mathrm{g}$ AG490 (an inhibitor of JAK-STAT) for $30 \mathrm{~min}$ prior to chronic morphine treatment, markedly attenuated the increased expression of spinal NR1 subunit induced by chronic morphine treatment, suggesting the spinal STAT pathway is involved in this upregulation process. AG490 $(1 \mu \mathrm{g})$ alone failed to alter the basal expression of spinal NR1 (Fig.6).

JAK-STAT inhibitor and NMDA receptor inhibitor block the development of morphine antinociceptive tolerance in rats. Finally, we determined the role of the JAK-STAT pathway 
A

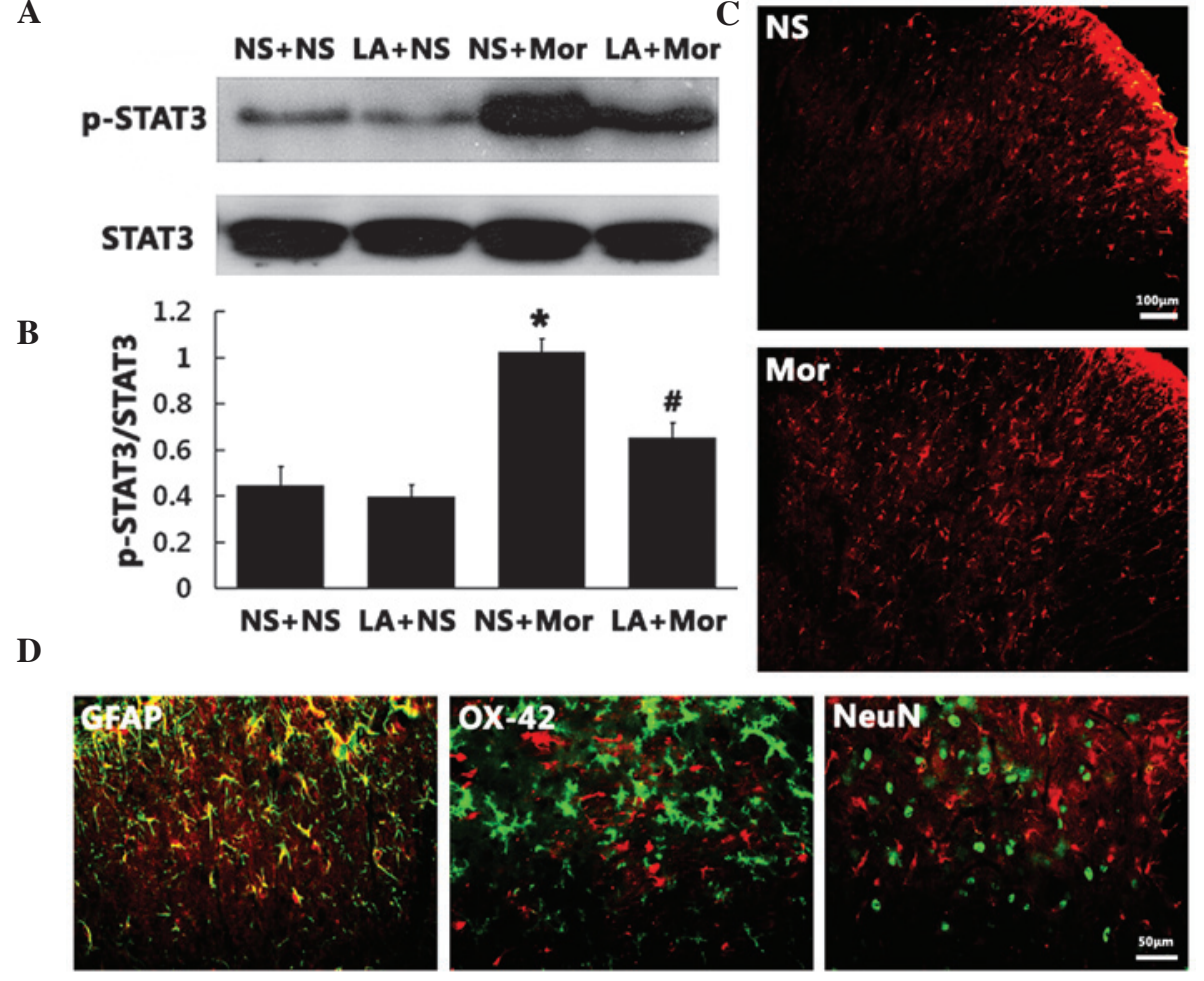

Figure 4. Spinal leptin contributes to chronic morphine-induced upregulation of spinal STAT3 expression in rats. LA ( $3 \mu \mathrm{g})$ was applied 30 min prior to the i.t. injection of Mor $(15 \mu \mathrm{g})$. (A) The expression of spinal STAT3 was detected by western blot analysis and (B) quantified by densitometric analysis of the data in (A) using Image J 1.41o software. (C) Immunofluorescence data demonstrated the increased p-STAT3 IR in the morphine injection group compared with the NS group. Scale bar: $100 \mu \mathrm{m}$. (D) Double-immunofluorescence results revealed that the p-STAT3 IR was colocalized with GFAP (an astroglia marker). Scale bar: $50 \mu \mathrm{m}$. Data are represented as the mean \pm SEM $(\mathrm{n}=3)$. ${ }^{*} \mathrm{P}<0.05$ vs. the NS+NS group, ${ }^{\prime} \mathrm{P}<0.05$ vs. LA+NS group. STAT, signal transducer and activator of transcription; GFAP, glial fibrillary acidic protein; NS, normal saline; LA, leptin antagonist; Mor, morphine; p, phosphorylated; IR, immunoreactivity.

A

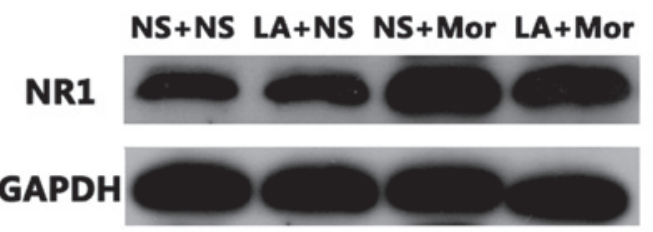

B

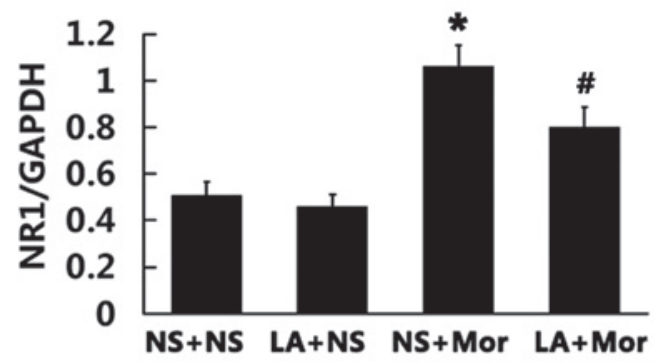

D

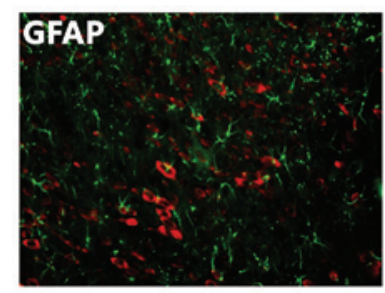

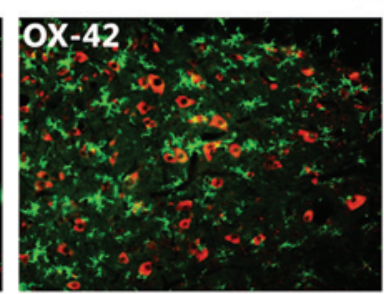
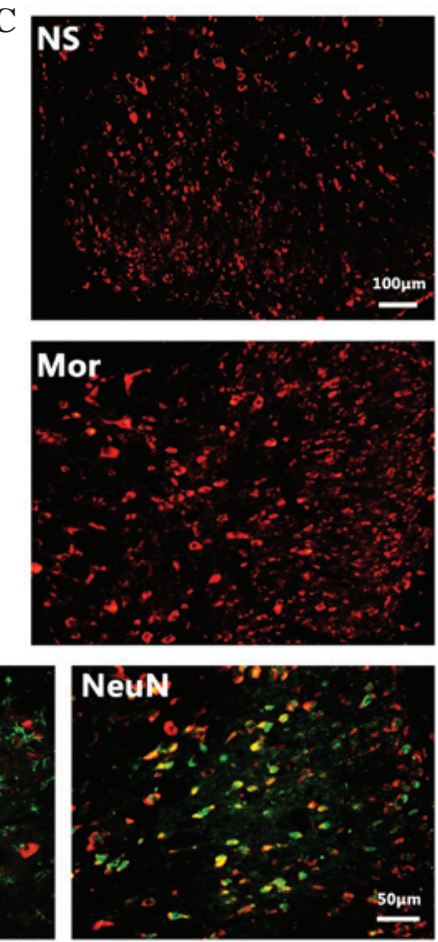

Figure 5. Spinal leptin modulates chronic morphine-induced increased expression of the spinal NR1 subunit of NMDA receptors in rats. LA ( $3 \mu \mathrm{g})$ was applied 30 min prior to the i.t. injection of Mor (15 $\mu \mathrm{g})$. (A) The expression of spinal NR1 was measured by western blot analysis and (B) quantified by densitometric analysis of the data in (A) using Image J 1.41o software. (C) The immunofluorescence data demonstrate that chronic Mor significantly increased the NR1 IR in the spinal cord dorsal horn compared with the saline (NS) group. Scale bar: $100 \mu \mathrm{M}$. (D) Double immunofluorescence staining results reveal that the spinal NR1 was colocalized with NeuN (a neuronal marker). Scale bar: $50 \mu$ M. Data are represented as the mean \pm SEM ( $n=3$ ). "P<0.05 vs. the NS+NS group, ${ }^{\#} \mathrm{P}<0.05$ vs. NS+Mor group. GFAP, glial fibrillary acidic protein; NMDA, N-methyl-D-aspartate; NS, normal saline; Mor, morphine; LA, leptin antagonist; i.t., intrathecal. 
A

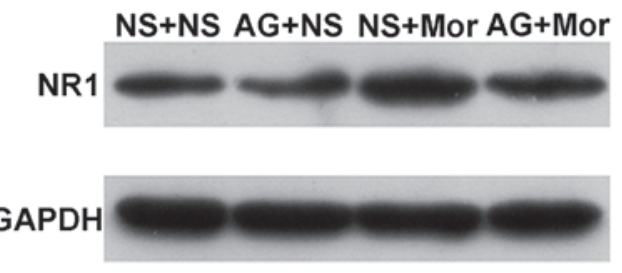

B

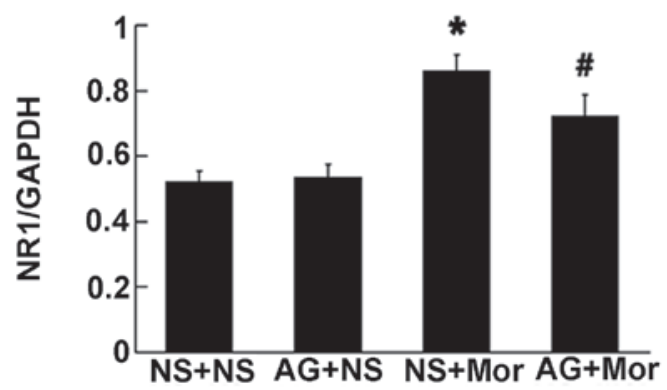

Figure 6. JAK-STAT pathway is involved in the upregulation of the expression of spinal NR1 subunit of NMDA receptors by chronic Mor in rats. (A) Western blotting revealed that the JAK-STAT pathway modulates the increased spinal NR1 expression following chronic Mor treatment. (B) The data in (A) were quantified by densitometric analysis using Image $\mathrm{J} 1.410$ software. Data are represented as the mean \pm SEM $(n=3)$. ${ }^{*} \mathrm{P}<0.05$ vs. NS+NS group, ${ }^{\#} \mathrm{P}<0.05$ vs. NS+Mor group. NMDA, N-methyl-D-aspartate; JAKSTAT, Janus kinase signal transducer and activator of transcription; NS, normal saline; Mor, morphine; AG, AG490 (an inhibitor of JAK-STAT).

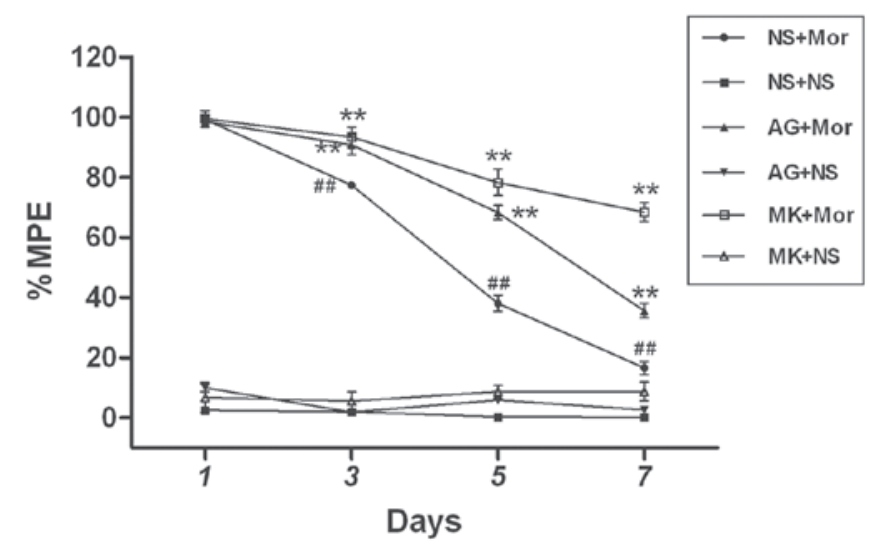

Figure 7. JAK-STAT inhibitor and NMDA receptor antagonist attenuate the development of Mor antinociceptive tolerance in rats. JAK-STAT inhibitor (AG490, $1 \mu \mathrm{g}$ ) or NMDA receptor antagonist (MK-801, $10 \mathrm{nM}$ ) was administered $30 \mathrm{~min}$ prior to daily Mor $(15 \mu \mathrm{g})$ i.t. injection. Data are presented as the mean \pm SEM $(n=6)$. ${ }^{* *} \mathrm{P}<0.001$ vs. (NS+Mor) group, ${ }^{\# \#} \mathrm{P}<0.001$ vs. (NS+ NS) group. NMDA, N-methyl-D-aspartate; JAK-STAT, Janus kinase signal transducer and activator of transcription; \%MPE, the percentage of maximal possible antinociceptive effect; NS, normal saline; Mor, morphine; AG, AG490; MK, MK-801.

and NMDA receptors in the spinal leptin-mediated development of morphine antinociceptive tolerance. As summarized in Fig. 7, pretreatment with either $1 \mu \mathrm{g}$ AG490 (an inhibitor of JAK-STAT) or $10 \mathrm{nM}$ MK-801, (a non-competitive antagonist of NMDA receptor) for $30 \mathrm{~min}$ prior to chronic morphine treatment, markedly blocked the development of morphine antinociceptive tolerance on days 3,5 and 7, as compared with the morphine treatment group, respectively. However, $1 \mu \mathrm{g}$ AG490 or $10 \mathrm{nM}$ MK-801 alone did not induce any analgesic effect as compared with the saline group (Fig. 7).
Combined with the above results of this study (Figs. 1, 3-6), these findings revealed that spinal leptin contributes to the development of morphine antinociceptive tolerance in rats, at least in part by activating the spinal STAT3-NMDA receptors pathway.

\section{Discussion}

In the present study, novel data were provided, demonstrating that spinal leptin contributes to the development of morphine antinociceptive tolerance through the activation of the STAT3-NMDA receptor pathway in rats. This is supported by the following findings: (i) chronic morphine treatment upregulated the expression levels of the spinal $\mathrm{Ob}-\mathrm{R}$ and leptin in a time-dependent manner; (ii) chronic morphine enhanced the spinal expression of p-STAT3; (iii) chronic morphine increased the spinal expression level of NR1 subunit of NMDA receptor; (iv) a LA blocked the development of tolerance to morphine analgesia; (v) chronic morphine-induced upregulation of the spinal expression of p-STAT3 and NR1 subunit of NMDA receptor was also attenuated by a LA; (vi) chronic morphine-induced upregulation of NR1 subunit expression was attenuated by AG490 (an inhibitor of JAK-STAT pathway) and (vii) AG490 and MK-801 (a non-competitive antagonist of NMDA receptor) reduced the development of morphine antinociceptive tolerance.

Leptin, the protein encoded by the obese (ob) gene, was originally considered to be an adipose-derived hormone (18). Recent studies, however, have reported that leptin is produced by a variety of tissues, including gastric mucosa, bone marrow, mammary epithelium, skeletal muscle (19) and the heart (20). In addition, leptin mRNA expression has also been identified in a variety of brain regions $(21,22)$. In the present study, we observed the expression of the spinal leptin protein and Ob-R expression, as well as their immunoreactivity, at a basal level in the spinal cord dorsal horn of rats. These data are consistent with previous results that there is a basal expression of the leptin mRNA and protein, as well as the long form of the leptin receptor $(\mathrm{Ob}-\mathrm{Rb})$, within the spinal cord dorsal horn (11). Based on previous investigations (11) and our studies, it was hypothesized that the spinal cord dorsal horn of rat is a site of leptin production and action.

Of note, leptin has been demonstrated to contribute to the pathogenesis of neuropathic pain (10-12). However, whether leptin is involved in the development of morphine antinociceptive tolerance is unclear. Since it is hypothesized that morphine tolerance and neuropathic pain share some common pathological mechanisms $(13,14)$, this prompted us to examine the role of spinal leptin in the development of morphine antinociceptive tolerance. The results demonstrated, for the first time, to the best of our knowledge, that the spinal leptin critically contributes to the development of morphine antinociceptive tolerance because (i) i.t. administration of a LA significantly attenuated the development of morphine antinociceptive tolerance; (ii) the expression of spinal leptin protein and immunoreactivity was markedly enhanced after chronic morphine treatment and (iii) the expression of spinal $\mathrm{Ob}-\mathrm{R}$ protein and immunoreactivity was evidently upregulated following chronic morphine treatment. However, the underlying mechanisms of the spinal leptin upregulation 
following chronic morphine remain elusive and further studies are required to investigate this.

To examine the molecular mechanisms underlying the role of spinal leptin in the development of morphine antinociceptive tolerance, we investigated whether the activation of STAT3 mediates spinal leptin-induced morphine antinociceptive tolerance. This was because activation of the JAK-STAT pathway has been demonstrated, via targeted gene transcriptions, to be responsible for a number of leptin functions, that were indicative of a genomic mechanism of action $(23,24)$. Leptin binds to the extracellular domain of the $\mathrm{Ob}-\mathrm{Rb}$ dimmer, activating the JAK2 tyrosine kinase. The activated JAK2 tyrosine kinase phosphorylates itself, Tyr1138 and Tyr1007 on the intracellular tail of Ob-Rb (17). The p-Tyr1138 binds and mediates the phosphorylation-dependent activation of STAT3. STAT3 then translocates to the nucleus to activate the transcription of downstream target genes. Recently, Lim et al reported that spinal leptin mediates neuropathic pain induced by chronic constriction sciatic nerve injury (CCI) by activating the JAK-STAT pathway, and that the i.t. leptin induced-pain behavioral and cellular changes (such as the upregulation of NR1 subunit of NMDA receptors and IL-1 $\beta$ expression) are markedly ameliorated by coadministration of AG490 (a JAK/STAT inhibitor) (11). In this study, consistent with a recent study reported by Wang et al (5), we demonstrated that the expression of spinal p-STAT protein (western blot analysis) and immunoreactivity, were significantly upregulated following chronic morphine treatment. The increased spinal p-STAT3 expression was attenuated by i.t. injection of a LA. In addition, pretreatment with AG490, prior to exposure to chronic morphine, markedly depressed the development of morphine antinociceptive tolerance. These results suggest that the activation of STAT3 is implicated in spinal leptin-mediated development of morphine antinociceptive tolerance, which extends the findings of a recent study (5).

NMDA receptors have been demonstrated to contribute to the pathogenesis of neuropathic pain $(11,12,25,26)$ and tolerance to morphine analgesia $(1,2,27)$. Furthermore, there appears to be a functional link between leptin and NMDA receptors. For example, exposure to leptin enhances NMDA receptor activity and modulates NMDA receptor-dependent long term potential (LTP) in an in vitro hippocampal preparation (28). I.t. administration of MK-801 attenuates exogenous leptin-induced thermal hyperalgesia and mechanical allodynia (12).

More recently, a study also demonstrated that leptin upregulates the expression of spinal NMDA receptor via the JAK/STAT pathway (11). Based on these previous studies $(1,2,11,12,25-28)$, we investigated the role of the NR1 subunit of the NMDA receptor in spinal leptin-mediated development of morphine antinociceptive tolerance. Our findings demonstrated that the activation of STAT3-NMDA receptor pathway is an important component of this process in rats. This is supported by the following results that (i) the level of spinal NR1 subunit protein and immunoreactivity was enhanced following chronic morphine administration, (ii) the increased expression of spinal NR1 was attenuated by a LA, (iii) the increased expression of spinal NR1 was diminished by AG490 (a JAK/STAT inhibitor) and (iv) MK-801, a non-competitive antagonist of NMDA receptor considerably blocked the development of morphine antinociceptive tolerance. However, a regulatory effect of descending neural pathways may not be excluded in our in vivo experiments. Therefore more studies, including in vitro experiments, are required to more accurately define whether the modulatory effects of spinal leptin on the activation of spinal STAT3-NR1 pathway were associated with its direct or indirect spinal action, or a combination of both.

To date, multiple factors have been demonstrated to contribute to the development of tolerance to morphine analgesia. Our results reveal that the spinal leptin-STAT3-NMDA pathway may be a unique target for new pharmacological interventions for patients exhibiting morphine tolerance, because leptin has been demonstrated to have a critical role in a number of neuronal functions, including neuroplasticity and neuroendocrine regulation (29). Further studies examining the role of other signaling cascades used by leptin, may facilitate our understanding of the mechanisms responsible for the contribution of leptin to the development of morphine antinociceptive tolerance.

\section{Acknowledgements}

This study was supported by grants from the Science and Technology Planning Project of Guangdong of China (2012B031800358) and the National Natural Science Foundation of China (no. 30800335 and no. 81100827).

\section{References}

1. Trujillo $\mathrm{KA}$ and Akil $\mathrm{H}$ : Inhibition of morphine tolerance and dependence by the NMDA receptor antagonist MK-801. Science 251: 85-87, 1991.

2. Guo RX, Zhang M, Liu W, Zhao CM, Cui Y, Wang CH, Feng JQ and Chen PX: NMDA receptors are involved in upstream of the spinal JNK activation in morphine antinociceptive tolerance. Neurosci Lett 467: 95-99, 2009.

3. Raghavendra V, Rutkowski MD and DeLeo JA: The role of spinal neuroimmune activation in morphine tolerance/hyperalgesia in neuropathic and sham-operated rats. J Neurosci 22: 9980-9989, 2002.

4. Johnston IN, Milligan ED, Wieseler-Frank J, Frank MG, Zapata V, Campisi J, Langer S, Martin D, Green P, Fleshner M, et al: A role for proinflammatory cytokines and fractalkine in analgesia, tolerance, and subsequent pain facilitation induced by chronic i.t. morphine. J Neurosci 24: 7353-7365, 2004.

5. Wang Z, Ma W, Chabot JG and Quirion R: Calcitonin gene-related peptide as a regulator of neuronal CaMKII-CREB, microglial p38-NFKB and astroglial ERK-Stat1/3 cascades mediating the development of tolerance to morphine-induced analgesia. Pain 151: 194-205, 2010.

6. Cui Y, Liao XX, Liu W, Guo RX, Wu ZZ, Zhao CM, Chen PX and Feng JQ: A novel role of minocycline: attenuating morphine antinociceptive tolerance by inhibition of p38 MAPK in the activated spinal microglia. Brain Behav Immun 22: 114-123, 2008.

7. Cui Y, Chen Y, Zhi JL, Guo RX, Feng JQ and Chen PX: Activation of p38 mitogen-activated protein kinase in spinal microglia mediates morphine antinociceptive tolerance. Brain Res 1069: 235-243, 2006.

8. Zhang Y, Proenca R, Maffei M, Barone M, Leopold L and Friedman JM: Positional cloning of the mouse obese gene and its human homologue. Nature 372: 425-432, 1994.

9. Bjørbaek C, Uotani S, da Silva B and Flier JS: Divergent signaling capacities of the long and short isoforms of the leptin receptor. J Biol Chem 272: 32686-32695, 1997.

10. Maeda T, Kiguchi N, Kobayashi Y, Ikuta T, Ozaki M and Kishioka S: Leptin derived from adipocytes in injured peripheral nerves facilitates development of neuropathic pain via macrophage stimulation. Proc Natl Acad Sci USA 106: 13076-13081, 2009.

11. Lim G, Wang S, Zhang Y, Tian Y and Mao J: Spinal leptin contributes to the pathogenesis of neuropathic pain in rodents. J Clin Invest 119: 295-304, 2009. 
12. Tian Y, Wang S, Ma Y, Lim G, Kim H and Mao J: Leptin enhances NMDA-induced spinal excitation in rats: A functional link between adipocytokine and neuropathic pain. Pain 152: 1263-1271, 2011.

13. Mao J, Price DD and Mayer DJ: Mechanisms of hyperalgesia and morphine tolerance: a current view of their possible interactions. Pain 62: 259-274, 1995.

14. Mayer DJ, Mao J, Holt J and Price DD: Cellular mechanisms of neuropathic pain, morphine tolerance, and their interactions. Proc Natl Acad Sci USA 96: 7731-7736, 1999.

15. Harvey J: Leptin: a diverse regulator of neuronal function. J Neurochem 100: 307-313, 2007.

16. Tartaglia LA, Dembski M, Weng X, et al: Identification and expression cloning of a leptin receptor, OB-R. Cell 83: 1263-1271, 1995.

17. Myers MG Jr: Leptin receptor signaling and the regulation of mammalian physiology. Recent Prog Horm Res 59: 287-304, 2004

18. Ahima RS, Qi Y, Singhal NS, Jackson MB and Scherer PE: Brain adipocytokine action and metabolic regulation. Diabetes 55 (Suppl 2): S145-154, 2006.

19. Wolsk E, Mygind H, Grøndahl TS, Pedersen BK and van Hall G: Human skeletal muscle releases leptin in vivo. Cytokine 60: 667-673, 2012.

20. Purdham DM, Zou MX, Rajapurohitam V and Karmazyn M: Rat heart is a site of leptin production and action. Am J Physiol Heart Circ Physiol 287: H2877-H2884, 2004.
21. Morash B, Li A, Murphy PR, Wilkinson M and Ur E: Leptin gene expression in the brain and pituitary gland. Endocrinology 140: 5995-5998, 1999.

22. Ur E, Wilkinson DA, Morash BA and Wilkinson M: Leptin immunoreactivity is localized to neurons in rat brain. Neuroendocrinology 75: 264-272, 2002.

23. Bjorbaek C: Central leptin receptor action and resistance in obesity. J Investig Med 57: 789-794, 2009.

24. Robertson SA, Leinninger GM and Myers MG Jr: Molecular and neural mediators of leptin action. Physiol Behav 94: 637-642, 2008.

25. Fisher K, Coderre TJ and Hagen NA: Targeting the N-methyl-D-aspartate receptor for chronic pain management. Preclinical animal studies, recent clinical experience and future research directions. J Pain Symptom Manage 20: 358-373, 2000.

26. Hewitt DJ: The use of NMDA-receptor antagonist in the treatment of chronic pain. Clin J Pain 16 (Suppl): s73-79, 2000.

27. Houghton AK, Parsons CG and Headley PM: Mrz 2/579, a fast kinetic NMDA channel blocker, reduces the development of morphine tolerance in awake rats. Pain 91: 201-207, 2001.

28. Goldin M, Segal M and Avignone E: Functional plasticity triggers formation and pruning of dendritic spines in cultured hippocampal networks. J Neurosci 21: 186-193, 2001.

29. Elmquist JK, Maratos-Flier E, Saper CB and Flier JS: Unraveling the central nervous system pathways underlying responses to leptin. Nat Neurosci 1: 445-450, 1998. 